\title{
UTILIZAÇÃO DO GPR PARA MAPEAMENTO NÃO DESTRUTIVO DE ESTRUTURA DE CONCRETO
}

\author{
Emerson Casagrande \\ Luiz Carlos de Almeida ${ }^{2}$ \\ Maria Teresa Françoso ${ }^{3}$ \\ Leandro Mouta Trautwein ${ }^{4}$ \\ Antõnio Carlos dos Santos ${ }^{5}$
}

\begin{abstract}
RESUMO
Ao longo dos anos, técnicas de ensaios não destrutivos foram desenvolvidas, visando o monitoramento e a avaliação das condições construtivas de estruturas de concreto armado. Na construção civil, as aplicações destes ensaios são para verificar as resistências à compressão, determinar o posicionamento e localização de vigas e pilares. Uma alternativa para investigação de estrutura de concreto armado é a utilização do sistema GPR (Ground Penetration Radar), que realiza a inspeção com leitura continua e de forma não destrutiva do elemento a ser estudado, tendo como resultado uma análise completa, sem a necessidade de interpolações. Pode localizar a posição ou determinar a profundidade de qualquer objeto incorporado à estrutura de concreto. Os alvos incluem armaduras, cabos, conduites, vazios, fraturas, etc.. 0 presente trabalho tem como objetivo mostrar estudo realizado em laboratório com o Ground Penetration Radar (GPR) como um método de ensaio não destrutivo na detecção de armaduras em estruturas de concreto armado e verificação de sua eficácia. Esse estudo foi realizado em uma placa de concreto com as dimensões e posicionamento das armaduras previamente conhecidas para validação do método. Foi utilizado o sistema GPR modelo SIR-3000 (GSSI) acoplado a uma antena de 1,6 GHz para a inspeção. 0 processamento dos dados coletados foram realizados no programa computacional RADAN (Radar Data Analyzer).

Palavras-chave: ensaio não destrutivos; estruturas de concreto; GPR.
\end{abstract}

\section{ABSTRACT}

Over the years, nondestructive methods have been developed, seeking the monitoring and evaluation of structures conditions of reinforced concrete structures. The applications of these methods are to verify the concrete strength and to determine the position and location of bars in beams and columns. An alternative to investigate reinforced concrete structures is the use of Ground Penetration Radar (GPR) which performs the inspection with continuing reading nondestructively the element to be studied, resulting in a complete analysis, without interpolations. It's

\footnotetext{
${ }^{1}$ Engenheiro Civil/ Mestrando - UNIVERSIDADE ESTADUAL DE CAMPINAS, CAMPINAS-SP, BRASIL polivalente2011@gmail.com

${ }^{2}$ Professor Doutor, Universidade Estadual de Campinas, Campinas-SP, Brasil. almeida@fec.unicamp.br ${ }^{3}$ Professora Doutor, Universidade Estadual de Campinas, Campinas-SP, Brasil.mtfrancoso@gmail.com ${ }^{4}$ Professor Doutor, Universidade Estadual de Campinas, Campinas-SP, Brasil. leandromt@fec.unicamp.br

${ }^{5}$ Professor Doutor, Universidade Federal de Uberlândia, UBERLÂNDIA-MG,BRASIL. acds@feciv.ufu.br
} 
possible to find the position or determine the depth of any object embedded in the concrete structure. The targets of this method include reinforcement, cables, conduits, voids, fractures, etc. To locate voids or fractures, it should be larger enough to be detected directly by the antenna. So the preventive maintenance of structures can be more efficiently and with low costs, which allows the extension of their useful life. The aim of the present work is to show a study in laboratory with the GRP (Ground Penetration Radar) as nondestructive method to detect the bars in reinforced concrete structures and its effectiveness. This study was realized on a concrete slab with the dimensions and positioning of reinforcement previously known to validate this method. In developing the work is used a model GPR SIR-3000 (GSSI) coupled to an antenna $1.6 \mathrm{GHz}$ for data collection. The processing and interpretation of the collected data is performed in software RADAN (Radar Data Analyzer).

Keywords: non-destructive testing; reinforced concrete structures; monitoring structures; GPR.

\section{INTRODUÇÃo}

A inspeção das armaduras na estrutura de um edifício ou uma obra de arte especial é uma tarefa difícil, visto que esta se encontra totalmente imersa em um meio opaco e rígido. Para este levantamento, os ensaios não destrutivos são os mais recomendados, pois não afetam a estrutura a qual a armadura compõe, são de baixo custo de execução não é necessário "rasgar" a camada de concreto que envolve a armadura e apresenta resultados rápidos.

O GPR (Ground Penetration Radar) é um método geofísico utilizado para a detecção de interferências em meios sólidos, principalmente no solo, na detecção de tubulações enterradas, contaminações. Nesse trabalho, o método geofísico será adaptado para pequenas profundidades (até $50 \mathrm{~cm}$ ) com a utilização de uma antena de alta resolução $(1,6 \mathrm{GHz})$ da fabricante Geophysical Survey Systems Incorporated (GSSI), acoplada ao sistema de aquisição de dados SIR3000.

\subsection{Ensaios não destrutivos}

Ensaios não destrutivos são aqueles que não causam qualquer dano ao elemento estudado, ou deixam pequenos danos que podem ser reparados após o ensaio. Segundo Lemos (2005), nos últimos 40 anos, várias tentativas têm sido feitas quanto ao desenvolvimento de métodos de ensaio "in situ" não destrutivos, para assegurar a qualidade do concreto na estrutura. Os métodos disponíveis podem ser classificados em:

a) métodos para determinar algumas propriedades do concreto que possibilitam uma estimativa de sua resistência, módulo de elasticidade e durabilidade; 
b) métodos onde são detectados posição e tamanho das armaduras, vazios, fissuras, falhas de concretagem, e teor de umidade do concreto "in loco".

Segundo Evangelista (2002), esses métodos são relevantes não só para o caso de estruturas executadas há algum tempo, que apresentam problemas e têm que ser reparadas e/ou reforçadas, mas também para o caso de estruturas novas ou ainda em execução (ensaios de aceitação). Dentre as propriedades do concreto que podem ser avaliadas por meio de ensaios não destrutivos, tem-se: massa específica, módulo de elasticidade e resistência. Inda pode ser investigada a dureza superficial, absorção, permeabilidade, condições de umidade, e também a existência de vazios, fissuração e localização das armaduras, objeto de estudo desse trabalho.

\subsection{Ground penetration radar (GPR)}

O GPR é um método de investigação geofísico proposto em 1904, pelo engenheiro alemão Christian Hülsmeyer, fundamentado na propagação de sinais eletromagnéticos para a localização remota de objetos enterrados no solo. Em 1910 ocorreu a primeira publicação descrevendo uma aplicação do método, feita por Gotthelf Leimbach e Heinrich Löwy, na Alemanha. Neste trabalho foi sugerido o uso de equipamentos que transmitiam ondas continuamente, porém foi só em 1926 que Hülsenbeck patenteou o primeiro equipamento que usava a emissão de pulsos de radar, com objetivo de investigar a natureza das estruturas em subsuperfícies. Em 1929, na Áustria, Stern realizou a primeira aplicação prática do GPR, com a finalidade de detectar espessuras de geleiras (PINTO, 2010).

Até meados da década de 70 as aplicações do GPR resumiam-se basicamente a determinar e mapear espessuras e fissuras em geleiras. Alguns trabalhos foram realizados na Antártica e no Ártico. Entre os quais se podem ser citados: Harrison, (1970), Campbell e Orange (1974) e Annan e Davis (1976).

Durante a Guerra do Vietnã, o exercito americano começou aprimorar o sistema GPR com o propósito de localizar túneis escavados e usados como rota de fuga pelos vietnamitas. Ainda durante a década de 70, o GPR foi usado pela National Aeronautics and Space Administration (NASA) em pesquisas da subsuperfície lunar, na missão Apollo 17, realizada em 11 de dezembro de 1972 (PINTO, 2010).

Nos estágios iniciais da aplicação do sistema GPR, um dos grandes empecilhos era o equipamento, pois era produzido artesanalmente, o que tornava mais difícil o acesso das universidades e empresas de pequeno porte ao equipamento. Só a partir de 1972, com a fundação da companhia GSSI, o equipamento passou a ser disponibilizado comercialmente (MOREY, 1974). Futuramente, surgiram novas empresas comerciais de tecnologia GPR, tais como, MALÅ Geoscience, PENETRADAR Corporation, IDSIngegneria dei Sistemi, 3D-RADAR, Sensors \& Software Inc., RADAR Systems Inc., entre outras.

Na década de 80, foi reconhecido como método de exploração geofísica por parte da comunidade geocientífica mundial. Segundo Porsani (1999), isso ocorreu devido ao 
desenvolvimento tecnológico, que possibilitou a construção de equipamentos com os modos de aquisição de registros digitais, otimizando sua portabilidade, baixando seu custo, tornando-se mais fácil sua aquisição. Com os avanços tecnológicos e após anos de estudos e aprimoramentos dos equipamentos, o GPR tornou-se um dos métodos mais eficazes para sondagens e investigações em pequenas profundidades (geofísica rasa), devido aos baixos custos e à praticidade na realização dos trabalhos de campo. Hoje o método possui uma grande variedade de aplicações, tais como: aplicações forenses, militares, engenharia civil, estudos de solos, exploração mineral, geologia básica e ambiental, geotecnia, hidrogeologia, pesquisa arqueológica, planejamento urbano, dentre outras.

\subsection{Fundamentos teóricos}

O sistema GPR, georadar, é fundamentado na propagação de ondas eletromagnéticas de altas frequências, na faixa de $10 \mathrm{MHz}$ a 2,5 GHz (DAVIS e ANNAN, 1989). Quando um pulso eletromagnético é emitido e atinge uma interface que separa propriedades eletromagnéticas diferentes, uma parcela da onda eletromagnética sofre reflexão e difração e a outra parcela continua propagando-se até ser completamente absorvida pelo meio. Parte da onda que foi refletida e difratada, em direção à superfície, é captada por antena receptora (Figura 1a). A antena receptora detecta os sinais e os envia para uma unidade de controle, onde é amplificado e armazenado em uma unidade de processamento de dados, gerando uma sequência de traços ou "scans" mostrados em registro como função do tempo de propagação (Figura 1b).

Figura 1 - Modo de aquisição (a) e registro de resultados (b)

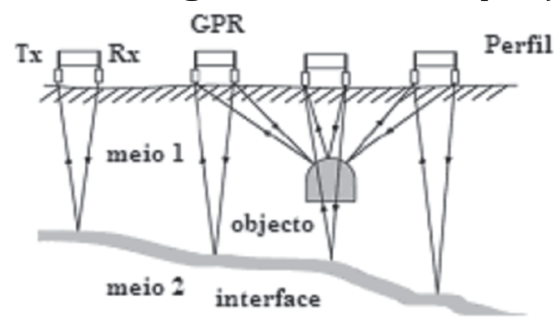

(a)

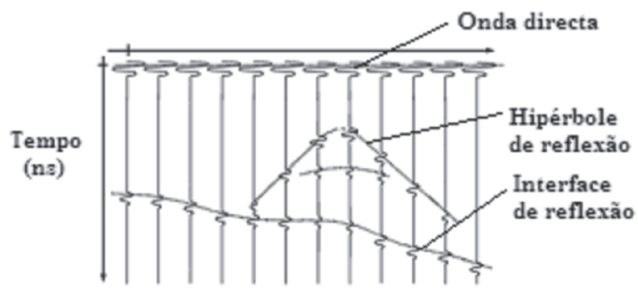

(b)

Fonte: Franco. 2010.

Quando a antena atravessa um alvo (tubo, cabo, vergalhões) em um ângulo reto, a imagem resultante se assemelha com uma hipérbole. Isso acontece porque a radiação do feixe da antena tem a forma de um cone, assim, o georadar pode ver o alvo não apenas quando em cima dele, mas também em várias verificações antes e depois dessa posição. A forma hipérbole revela a antena se aproximando do alvo e, em seguida, se distanciando. 0 cume da hipérbole mostra exatamente a posição do alvo (figura 2).

A representação dos scans obtidos ao longo de um perfil fornece as seções 2D denominadas radargramas. 
_Figura 2 - localização de um alvo.

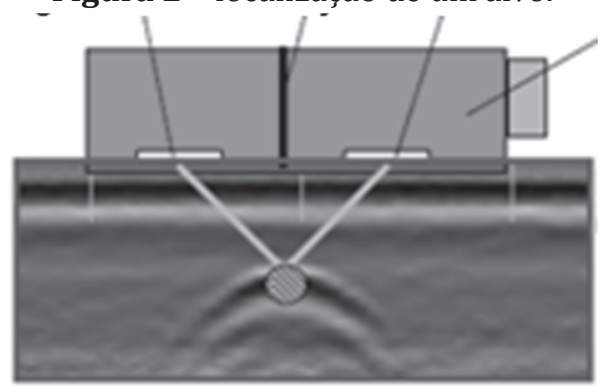

Fonte: (GSSI. 2009)

\section{PROBLEMA ANALISADO}

A primeira etapa do trabalho consistiu em uma revisão bibliográfica envolvendo os métodos geofísicos utilizados e leitura dos manuais de operação dos equipamentos. Em seguida, preparou-se um bloco de concreto armado com dimensões conhecidas e armaduras posicionadas em pontos conhecidos para realização da inspeção não destrutiva com o georadar.

A peça apresenta as dimensões de $85 \times 21 \times 16 \mathrm{~cm}$, sendo composta de uma fôrma de chapas de compensado com espessura de $1,5 \mathrm{~cm}$. No seu interior foram colocadas oito armaduras, além de 2 tubos de PVC com diâmetros nominais de $1 / 2$ " e $3 / 4$ " e uma esfera vazia de diâmetro 11/2", dispostos como apresentado nas Figuras 3, 4 e 5.

Figura 3 - Representação 3D da peça de estudo

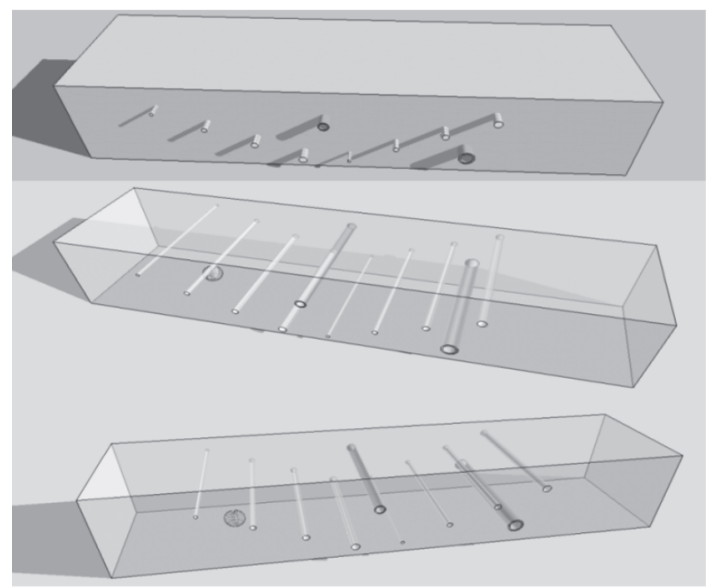

Fonte: Autor 
Figura 4 - Cortes superior e lateral da caixa e posição das armaduras e tubos
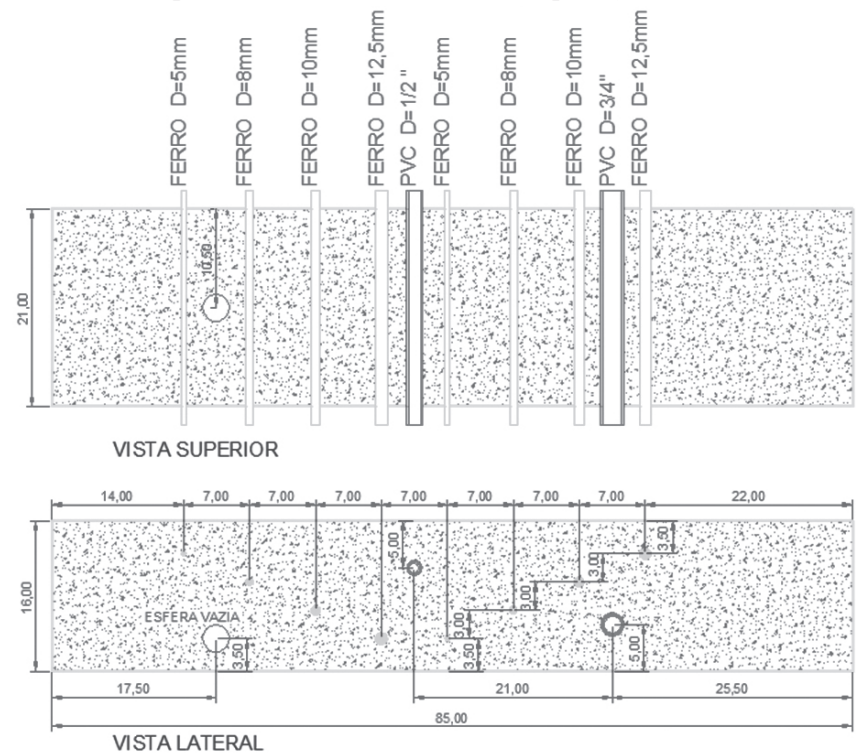

Fonte: Autor

Figura 5 - Posicionamento das interferências no bloco de concreto

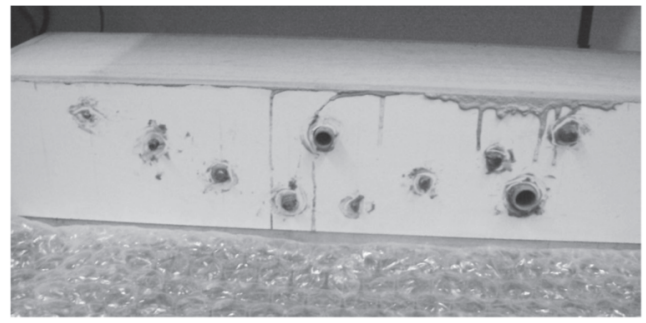

Foram realizadas varrições com o georadar no sentido longitudinal do bloco com o intuito de se obter as armaduras transversalmente, variando parâmetros de aquisição de dados do sistema GPR para a verificação das alterações causadas no radargrama obtido.

O sistema GPR da GSSI - SIR3000 apresenta seis modos, com configurações prédefinidas, para a realização do escaneamento em diversos locais para determinados fins. São eles (GSSI, 2011a):

a) TerraSIRch - permite que sejam determinados todos os parâmetros para a coleta dos dados para qualquer tipo de antena e finalidade de investigação;

b) 3Dscan - semelhante ao modo TerraSIRch, porém com a característica 
adicional de facilitar a coleta de dados 3D;

c) ConcreteScan - utilizado para uma varredura rápida em estruturas de concreto, para a localização de características rasas e determinação direta antes de executar um corte na estrutura, por exemplo. Nesse modo, não é possível realizar o processamento no software;

d) StructureScan - também utilizado em estruturas de concreto, permite que sejam coletados dados mais complexos que no modo ConcreteScan, sendo possível realizar uma varredura $3 \mathrm{D}$, com a ajuda de uma malha quadriculada;

e) UtilityScan - empregado para um exame rápido sobre uma área sob lajes de concreto ou asfalto. Utilizado com antenas de média resolução, permite que seja marcada a localização de interferências antes da abertura de valas ou escavação;

f) GeologyScan - modo otimizado para a criação de perfis 2D de características geológicas rasas.

Para esse estudo, o modo StrutureScan foi o que apresentou melhor resultado, sendo possível fazer uma leitura satisfatória dos resultados no radargrama.

\subsection{Procedimento dos levantamentos}

Para a execução dos testes foi utilizada a antena da GSSI de 1,6 GHz (Figura 6) de propriedade da Faculdade de Engenharia Civil da Universidade Estadual de Campinas (FEC - UNICAMP).

Figura 6 - Antena GSSI de $1.6 \mathrm{GHz}$.

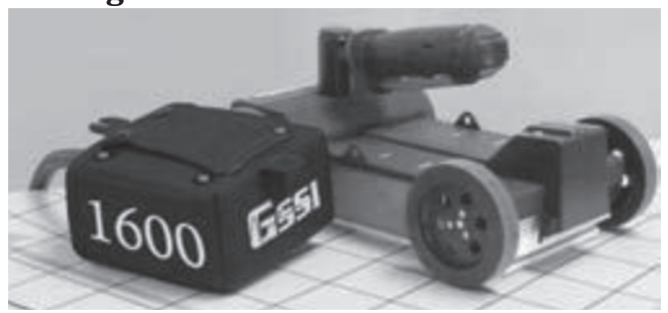

Fonte: GSSI. 2007

As varreduras realizadas no bloco foram executadas a partir de $15 \mathrm{~cm}$ da borda para acomodar o carrinho da antena e detectar todas as armaduras posicionadas no interior da peça, como pode se verificar na Figura 7. 
Figura 7 - Posicionamento da antena para varredura

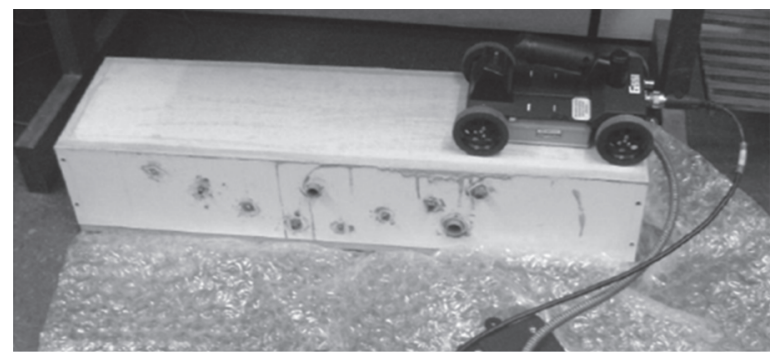

Fonte: Autor

\section{RESULTADOS}

As figuras 8 e 9 apresentam o radargrama obtido na investigação realizada, com aquisição dos dados no modo StrutureScan, do sistema GPR.

$\mathrm{Na}$ Figura 8, pode-se perceber pontos mais claros os quais correspondem ao posicionamento dos elementos colocadas no modelo estudado (Figuras 4 e 5). Ao se comparar as hipérboles formadas pelas armaduras com as formadas ao se encontrar a tubulação de PVC, nota-se que são mais fortes. Isso acontece, pois as ondas que atingem a superfície do aço refletem muito mais que aquelas que chegam aos PVCs, formando uma imagem mais "nítida". Com um pouco de dificuldade de interpretação das hipérboles, é possível identificar o vazio. Uma linha de picos pretos indica a existência de ar, onde pode ser identificado como sendo o fundo do concreto.

Figura 8 - Radargrama obtido da amostra

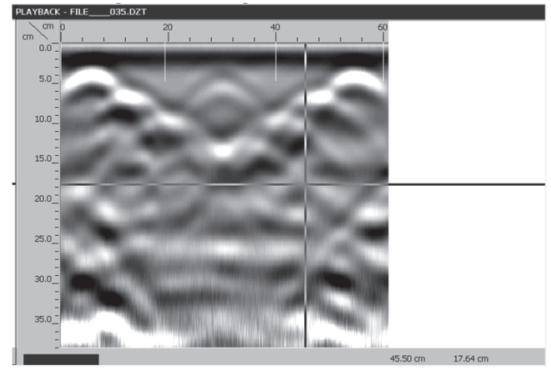

Fonte: Autor

Na Figura 9, ao se tomar o pico da hipérbole e verificar as distâncias em relação ao topo da peça, têm-se as alturas em que se encontram as armaduras. Os espaçamentos entre os alvos também são verificados com certa precisão, 'fornecendo resultados bastante satisfatórios. 
Figura 9 - Localização e identificação dos alvos

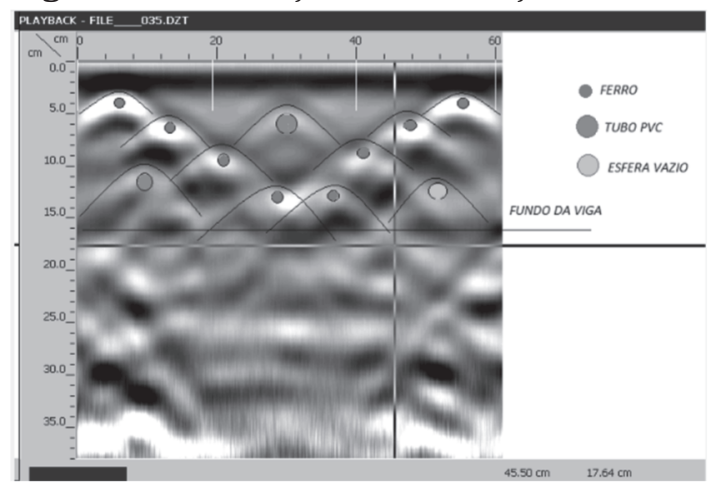

Fonte: Autor

Os dados coletados com o sistema GPR da GSSI foram processados no Programa RADAN ${ }^{\circledR}$, cuja tela de processamento está apresentada na Figura 10, para se obter imagens tridimensionais da estrutura em estudo, bem como as dimensões dos alvos.

Figura 10 - Processamento da varredura adquirida no programa RADAN

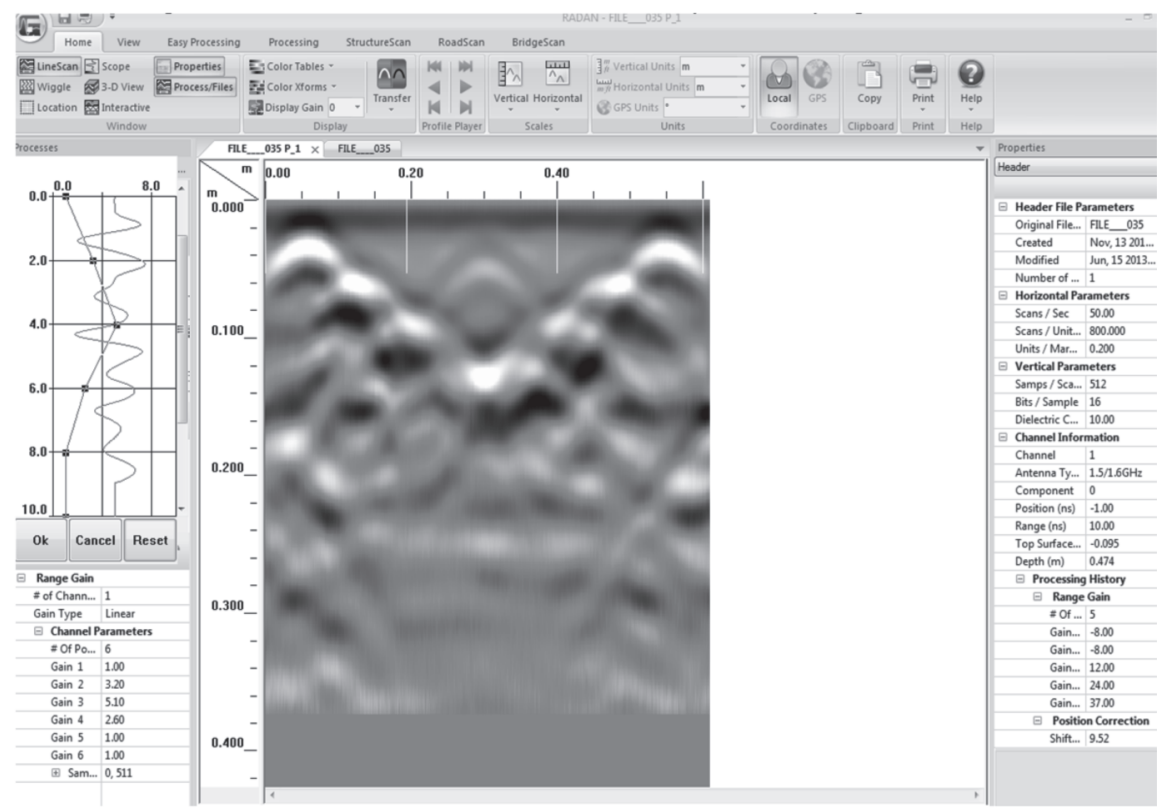




\section{CONCLUSÕES}

Nesse trabalho foi possível verificar também a diferença no sinal existente entre barras de aço e materiais de PVC, podendo ser útil para obtenção de "as built" e criação de arquivos de edifícios antigos, onde não é possível obter a documentação original dos projetos.

Apresenta vantagens sobre os outros métodos pela facilidade de execução e rapidez na obtenção dos resultados, porém, necessita de um profissional com grande experiência em trabalhos distintos e conhecimento dos princípios físicos e matemáticos que envolvem o método para interpretação dos alvos no radargrama, bem como no aproveitamento de todos os recursos oferecidos pelos softwares de interpretação existentes no mercado.

Uma proposta de estudos futuros é relacionar as propriedades geométricas da hipérbole com o diâmetro das barras para determinação das bitola sem que haja a necessidade de métodos invasivos na estrutura.

\section{AGRADECIMENTOS}

Os autores agradecem a Coordenação de Aperfeiçoamento de Pessoal de Nível Superior pela aquisição do sistema GPR através do Programa PRÓ-EQUIPAMENTOS INSTITUCIONAL da CAPES, em 2010.

\section{REFERÊNCIAS}

ANNAN, A. P.; DAVIS, J. L. Impulse radar sounding in permafrost. Radio Science, v. 11, n. 4 , p. 383-394, 1976.

CAMPBELL, K. J.; ORANGE, A. S. A Continuous profile of sea ice and freshwater ice thickness by impulse radar. Polar Record, v. 17, n. 106, p. 31-41, January 1974.

\section{DAVIS, J. L.; ANNAN, A. P. Ground-penetration radar for high-resolution} mapping of soil and rock stratigraphy. Geophysical Prospecting, v. 37, p. 531$551,1989$.

EVANGELISTA, A. C. J. Avaliação da Resistência do Concreto Usando Diferentes Ensaios não Destrutivos. Tese de doutorado. Universidade Federal do Rio de Janeiro. Rio de Janeiro, p. 219. 2002.

\section{FRANCO, D. A. L. Avaliação das potencialidades do georadar em Engenharia} Civil. Universidade de Aveiro. Aveiro, p. 150. 2010.

GSSI. Site da empresa: Alpha Geofísica Brasil. GSSI Concrete Antennas de 2600 a 1000MHz, 2007. Disponível em: 
<http://www.alphageofisica.com.br/gssi/GPR_2009/GSSI-

ConcreteAntennas_de_2600_a_1000Mhz_br.pdf >. Acesso em: 05 Agosto 2012.

GSSI. SIR System-3000. Salem: Geophysical Survey Systems, Inc., 2011a.

GSSI. UtilityScan: Quick Start Guide. [S.l.]: Geophysical Survey System, Inc.,2010.

GSSI. Handbook for RADAR inspection of Concrete: Geophysical Survey System, Inc., 2009.

HARRISON, C. Reconstruction of subglacial relief from radio echo sounding records. Geophysics, v. 35, n. 6, p. 1109-1115, December 1970.

LEMOS, K. B. Q. Manutenção e Reabilitação de Túneis. Universidade de Brasília. Brasília, p. 186. 2005.

MOREY, R. M. Continuous subsurface profiling by impulse radar. Subsurface Exploration for Underground Excavation and Heavy Construction. Henniker: American Society of Civil Engineers. 1974. p. 213-232. 39

PINTO, G. P. 0 Método GPR aplicado a localização de tubulações utilizadas no abastecimento de água na região urbana do município de Belém Pará. Dissertação. UFPA - 2010

PORSANI, J. L., 1999, Ground Penetrating Radar (GPR): Proposta Metodológica de Emprego em Estudos Geológico-geotécnicos nas Regiões de Rio Claro e Descalvado -SP. Tese de Doutorado, Instituto de Geociências e Ciências Exatas, UNESP, Campus de Rio Claro - SP. 145p. 